INFLAMMATION

\title{
Colonic epithelial cell mediated suppression of CD4 T cell activation
}

\author{
S M Cruickshank, L D McVay, D C Baumgart, P J Felsburg, S R Carding
}

Gut 2004;53:678-684. doi: 10.1136/gut.2003.029967

See end of article for authors' affiliations

.....................

Correspondence to: Dr S Cruickshank, School of Biochemistry and Molecular Biology, University of Leeds, Leeds LS2 9JT, UK; bmbsmc@ bmb.leeds.ac.uk

Accepted for publication 14 November 2003

\begin{abstract}
Background and aims: As the first point of contact with enteric antigens, intestinal epithelial cells (IEC) may be key in regulating mucosal immune responses. We determined therefore if murine colonic epithelial cells (CEC) have tolerogenic or activating effects on CD4 T cells.

Methods: Using a novel CEC, macrophages, and CD4 T cell coculture system, mitogen and antigen specific responses of naïve and antigen primed CD4 T cells were assessed.

Results: Although a proportion of CEC express the costimulatory molecules B7.1, B7.2, CD40, and CD54, they were unable to promote mitogen or antigen driven activation of CD4 T cells, even in the presence of exogenous costimulatory signals. CD4 T cells cocultured with CEC were CD25 $5^{\text {lo }}$ and CD $45 R^{\text {lo }}$ and remained in the G1 phase of the cell cycle. CEC were also able to prevent CD4 T cell activation by professional antigen presenting cells. CEC mediated suppression of T cell activation was cell contact dependent and transforming growth factor $\beta$ independent.

Conclusions: These observations suggest that CEC contribute to the maintenance of T cell tolerance in the gut by preventing inappropriate activation of CD4 T cells.
\end{abstract}

for ntestinal epithelial cells (IEC) are a major point of contact for enteric antigens and may play a direct role in mucosal immunity, particularly in regulating $\mathrm{T}$ cell responses to enteric antigens. ${ }^{1}$ The ability of IEC to constitutively or inducibly express molecules involved in antigen presentation, including major histocompatibility complex (MHC) class I and class II and the MHC I-like (MHC Ib, MIC-A, MIC-B, HLA-E, CDld) molecules, and to take up and process soluble protein antigens and secrete cytokines, suggests they can function as antigen presenting cells (APC). ${ }^{1-7}$ Furthermore, IEC express elevated levels of MHC II in human inflammatory bowel disease (IBD) $)^{2}$ and in animal models of $\mathrm{IBD}^{8}$ consistent with their involvement in the development and maintenance of IBD.

Much of the work investigating the interactions of IEC with $\mathrm{T}$ cells has focused on small IEC. However, the architecture, function, and antigenic load of the colon and small intestine are very different, including the composition of resident $\mathrm{T}$ cells. Whereas $\mathrm{T}$ cells in the small intestine are largely $\mathrm{CD}^{+} \alpha \beta$ or $\gamma \delta$ intraepithelial lymphocytes, the majority of $\mathrm{T}$ cells in the colon are epithelia associated $\mathrm{CD}^{+} \alpha \beta$ T cells. In the murine small intestine, specialised epithelial cells known as $M$ cells take up and transport antigen and may act as APC'; by comparison, little is known of professional APC within the colonic lamina propria. Our recent work demonstrating that murine colonic epithelial cells (CEC) have properties of $\mathrm{APC}^{10}$ suggest that they may be colonic APC.

$\mathrm{T}$ cell activation requires two major signals from an APC. Antigen must be presented to the $\mathrm{T}$ cell receptor (TcR) complex in the context of a MHC molecule (signal 1). Signal 2 involves interaction of a costimulatory molecule on the APC with its counter ligand on the $\mathrm{T}$ cell. Interaction of members of the B7 family of proteins (for example, B7.1, B7.2) with their counter receptors CD28 and CTLA-4 on T cells is important in $\mathrm{T}$ cell activation and suppression, respectively. ${ }^{11}$ Most normal epithelial cells such as biliary epithelial cells and hepatocytes do not express B7.1 or B7.2 but express other costimulatory molecules, including CD40 and CD54 (ICAM-1). ${ }^{12-14}$ We and others have demonstrated that epithelial cells such as biliary and lung epithelial cells lacking expression of B7 inhibit T cell activation. ${ }^{15-17}$ As activation of $\mathrm{T}$ cells in the absence of $\mathrm{B} 7$ causes $\mathrm{T}$ cell non-responsiveness or anergy and the majority of normal epithelial cells lack B7, it might be predicted that normal epithelial cells anergise $\mathrm{T}$ cells. The capacity of epithelial cells in the gastrointestinal tract to express B7.2 in response to infection ${ }^{13}$ or inflammation $^{18}{ }^{19}$ may provide a mechanism for CD4 $\mathrm{T}$ cell mediated immune response in colitis.

In this study, we have investigated the nature of T cell:CEC interactions and determined if CEC, in common with a number of other epithelial cell types, can prevent $\mathrm{T}$ cell activation.

\section{METHODS \\ Animals}

C57BL/6 and BALB/C-DO11.10 T cell receptor transgenic mice were purchased from Jackson Laboratory (Bar Harbor, Maine, USA) and housed under specific pathogen free conditions at the University of Leeds. Sentinel mice were routinely screened for common mouse pathogens and none was detected.

Isolation and culture of CEC and lamina propria T cells We have previously described the isolation and culture of CEC and lamina propria T cells. ${ }^{20} \mathrm{CEC}$ purity was assessed by staining for cytokeratin and CD45. Isolated CEC routinely comprise crypts, enterocytes, and goblet cells. Under our normal culture conditions, clusters of crypt-like cells, or organoids, attach to the Matrigel substrate and form semiconfluent monolayers of defined polarity with the epithelial

Abbreviations: APC, antigen presenting cells; $C B A$, cytometric bead array; CFSE, carboxyfluorescein diacetate succinimidyl ester; CEC, colonic epithelial cells; $F B S$, fetal bovine serum; IBD, inflammatory bowel disease; IEC, intestinal epithelial cells; IFN- $\gamma$, interferon $\gamma$; IL, interleukin; LAP, latency associated peptide; LP, lamina propria; MF, median fluorescence; MHC, major histocompatibility complex; PMA, phorbol12-myristate 13-acetate; TcR, T cell receptor; TGF- $\beta$, transforming growth factor $\beta$; TNF- $\alpha$, tumour necrosis factor $\alpha$ 
basolateral surface in contact with the Matrigel. However, in coculture with CD4 T cells, organoids remained intact and in suspension, allowing CD4 $\mathrm{T}$ cells to interact with both the basal and apical epithelial surfaces. CEC were retrieved from culture by digestion with dispase:collagenase IV (1 mg/ml; Sigma, Poole, Dorset, UK) for 5-10 minutes, after which cells were washed twice in Dulbecco's modified Eagle's medium containing $20 \%$ fetal bovine serum (FBS).

\section{CEC:CD4 T cell coculture}

Splenic CD4 T cells were prepared by depleting $\mathrm{M} \phi$ by plastic adherence, followed by negative immunomagnetic selection (Miltenyi Biotec, Bisley, UK) to produce a cell suspension containing $>98 \%$ CD4 $\mathrm{T}$ cells. Colonic lamina propria CD4 cells were isolated as described previously. ${ }^{20}$ For proliferation assays, T cells were labelled with carboxyfluorescein diacetate succinimidyl ester (CFSE; Cambridge Bioscience, Cambridge, UK) immediately prior to adding to CEC cultures. CEC organoids (approximately $2 \times 10^{5}$ cells $/ \mathrm{ml}$ ) were cultured for 6-24 hours before addition of whole spleen cells or CD4 T cells $\left(2 \times 10^{6} / \mathrm{ml}\right)$ in the presence or absence of ConA $(5 \mu \mathrm{g} /$ $\mathrm{ml}$; Sigma), mitogenic anti-CD3 antibody (1452C11), antiCD3 and anti-CD28 (37.51) antibodies, or OVA (10 $\mu \mathrm{M}$; Sigma). As a positive control, CD4 cells were cultured with adherent splenic $\mathrm{M} \phi\left(2 \times 10^{5} \mathrm{cells} / \mathrm{ml}\right)$ in Matrigel coated tissue culture plates (Becton Dickinson Biosciences, Oxford, UK) under the same conditions as CEC:CD4 T cell cocultures. Lamina propria $\mathrm{T}$ cell:CEC cocultures were performed as described above in the presence of mitogenic anti-CD3 antibody. In some experiments, $200 \mathrm{U} / \mathrm{ml}$ recombinant murine interleukin 2 (IL-2; TOTAM Biologicals, Cambridgeshire, UK), goat anti- transforming growth factor $\beta 1$ (TGF- $\beta 1$ ) antibodies, or goat IgG (R\&D Systems, Abingdon, UK) were added to CEC:T cell cocultures. T cells were harvested after 72 hours and analysed by antibody staining and flow cytometry.

\section{Antigen specific CD4 T cell responses}

Antigen primed CD4 $\mathrm{T}$ cells were obtained by culturing DO- 11 CD4 T cells of which $>90 \%$ expressed the OVA specific $\mathrm{TcR}\left(\mathrm{KJ} 1-26^{+}\right)$for five days in the presence of $10 \mu \mathrm{M}$ OVA and $20 \mathrm{U} / \mathrm{ml} \mathrm{IL-2} \mathrm{in} \mathrm{complete} \mathrm{RPMI} \mathrm{medium} \mathrm{(10 \%} \mathrm{v/v} \mathrm{FBS,}$ $100 \mathrm{U} / \mathrm{ml}$ penicillin, $100 \mu \mathrm{g} / \mathrm{ml}$ streptomycin, $2 \mathrm{mM}$ L-glutamine) at a final concentration of $2 \times 10^{6}$ cells $/ \mathrm{ml}$. Primed CD4 $\mathrm{T}$ cells were then isolated by positive immunomagnetic selection and cultured with CEC or $\mathrm{M} \phi$ in the presence or absence of OVA $(10 \mu \mathrm{M})$ for 72 hours prior to analysis. Freshly isolated non-stimulated DO11 CD4 cells were used as a source of naïve $\mathrm{T}$ cells in CEC or $\mathrm{M} \phi$ cocultures. As an additional control, FITC-OVA pulsed CEC were cultured with the lacZ-inducible OVA/I-A ${ }^{\mathrm{b}}$ specific CD4 hybridoma KZO, as described elsewhere. ${ }^{20}$

\section{Restimulation of CD4 T cells after coculture with CEC}

CEC and CD4 $\mathrm{T}$ cells were cocultured in the presence of mitogenic anti-CD3 antibody. After 72 hours, CD4 cells were isolated by positive immunomagnetic selection and restimulated with anti-CD3 antibody for 72 hours, or with $10 \mathrm{nM}$ phorbol-12-myristate 13-acetate (PMA) and $500 \mathrm{nM}$ ionomycin (CN Biosciences, Nottingham, UK) for 24 hours, as described previously. ${ }^{17} \mathrm{~T}$ cell activation was assessed by cell cycle analysis and expression of activation markers. The ability of OVA primed CD4 T cells to respond to antigen after coculture with CEC was also assessed. CD4 T cells were isolated from initial CEC:T cell cocultures by Percoll density gradient centrifugation, washed, and plated onto antigen pulsed $\mathrm{M} \phi$ for 72 hours prior to analysis.

\section{Flow cytometry}

Cells were analysed by flow cytometry on a FACScan flow cytometer using CELLQuest software (Becton Dickinson). Cells were stained with fluorochrome and/or biotin conjugated antibodies to CD45, B7.1 (CD80), B7.2 (CD86), ICAM-1 (CD54), CD40, KJ1-26, CD25, CD69, H-2D ${ }^{b}$, CD45RB, CD62L, CD44, CD8 $\alpha$, CD4, NK1.1, GR-1, F4/80 (TCS Biologicals, Towcester, UK), Fas (CD95), CTLA-4 (CD152), I-A/I-E (Becton Dickinson), a pan-reactive anticytokeratin-FITC antisera (Sigma), CD3 (1452C11; ATCC, Rockville, Maryland, USA), CD28 (clone 37.51; Dr M Glennie, Southampton, UK), goat antihuman latency associated peptide of TGF- $\beta 1$ (LAP), chicken antihuman TGF- $\beta$, and chicken IgY ( Stratech, Cambridgeshire, UK). Isotype matched antibodies of irrelevant specificity were used to determine the level of non-specific staining and frequency of cells stained with test antibodies. ${ }^{20}$ Apoptotic cells were identified by labelling cells with FITC-annexin $\mathrm{V}$ and propidium iodide. For intracellular staining, cells were surface stained, fixed in $0.1 \%$ paraformaldehyde, and permeablised with $0.1 \%$ saponin/1\% milk protein prior to staining. For intracellular cytokine staining, freshly isolated cells were incubated with Brefeldin A $(10 \mathrm{mg} / \mathrm{ml})$ for five hours prior to staining, as described above. For cell cycle analysis, propidium iodide was added immediately prior to acquisition. Cytokine multiplex bead assay kits (Becton Dickinson) were used to measure levels of interferon $\gamma$ (IFN- $\gamma$ ), tumour necrosis factor $\alpha$ (TNF$\alpha$ ), interleukin (IL)-5, IL-4, and IL-10 in CEC:T and M $\phi$ :T cell conditioned media.

\section{Statistics}

Data are presented as means (SEM). Statistical significance was evaluated using a Mann-Whitney test.

\section{RESULTS}

\section{CEC inhibit T cell activation by mitogens}

In the presence of $\mathrm{M} \phi$, colonic lamina propria (LP) and splenic CD4 T cells underwent blast formation and acquired the large granular morphology characteristic of activated cells. A significant proportion of splenic and lamina propria CD4 T cells were in S and G2 phases of the cell cycle (fig 1D, E) and proliferating as determined by CFSE staining of which splenic $\mathrm{T}$ cells had the response of greatest magnitude. Expression of activation associated surface antigens CD69 and CD25 (fig 1A, C) was induced or upregulated (CD95 (Fas), CD44, and CD45RB; fig 1B) on CD4 T cells.

In contrast, the response of both $\mathrm{LP}$ (fig $\mathrm{IC}, \mathrm{E}$ ) and splenic CD4 cells (fig 1A, B, D, F, and G) to activation in the presence of CEC was inhibited. The majority of CD4 cells were small and quiescent, with $>90 \%$ remaining in the Gl phase of the cell cycle (fig 1D, E). Few of the CD4 T cells in CEC coculture proliferated (data not shown) and the majority were CD25 $25^{-1 \mathrm{o}}$ $(\mathrm{p}<0.001$; fig $\mathrm{lA}, \mathrm{G})$ and expressed lower levels of CD45RB $(\mathrm{p}<0.001$; fig $1 \mathrm{~B})$ and CD95 $(\mathrm{p}<0.01)$ compared with CD4 T cells cultured with $\mathrm{M} \phi$. CEC also affected $\mathrm{T}$ cell cytokine production, and concentrations of IL-10, IL-5, IL-4, IFN- $\gamma$, and TNF- $\alpha$ were considerably reduced in the supernatants of CEC:CD4 $\mathrm{T}$ cell cocultures compared with $\mathrm{M} \phi$ :CD4 $\mathrm{T}$ cell cocultures (fig $1 F, G$ ). Using the CBA analysis system (detection limit of 10-20 pg), it was not possible to detect the presence of any of these cytokines in supernatants from CEC cultured in complete media alone (data not shown). Using intracellular staining with anticytokine antibodies, freshly isolated CD4 T cells expressed no detectable IFN- $\gamma$, TNF- $\alpha$, IL-2, IL-4, or IL-10 (data not shown).

As LP $\mathrm{T}$ cells are relatively refractory to stimulation through their $\mathrm{TcR}^{21}$ making measurement of normal indices of $\mathrm{T}$ cell function difficult, splenic CD4 T cells were used in subsequent experiments. 

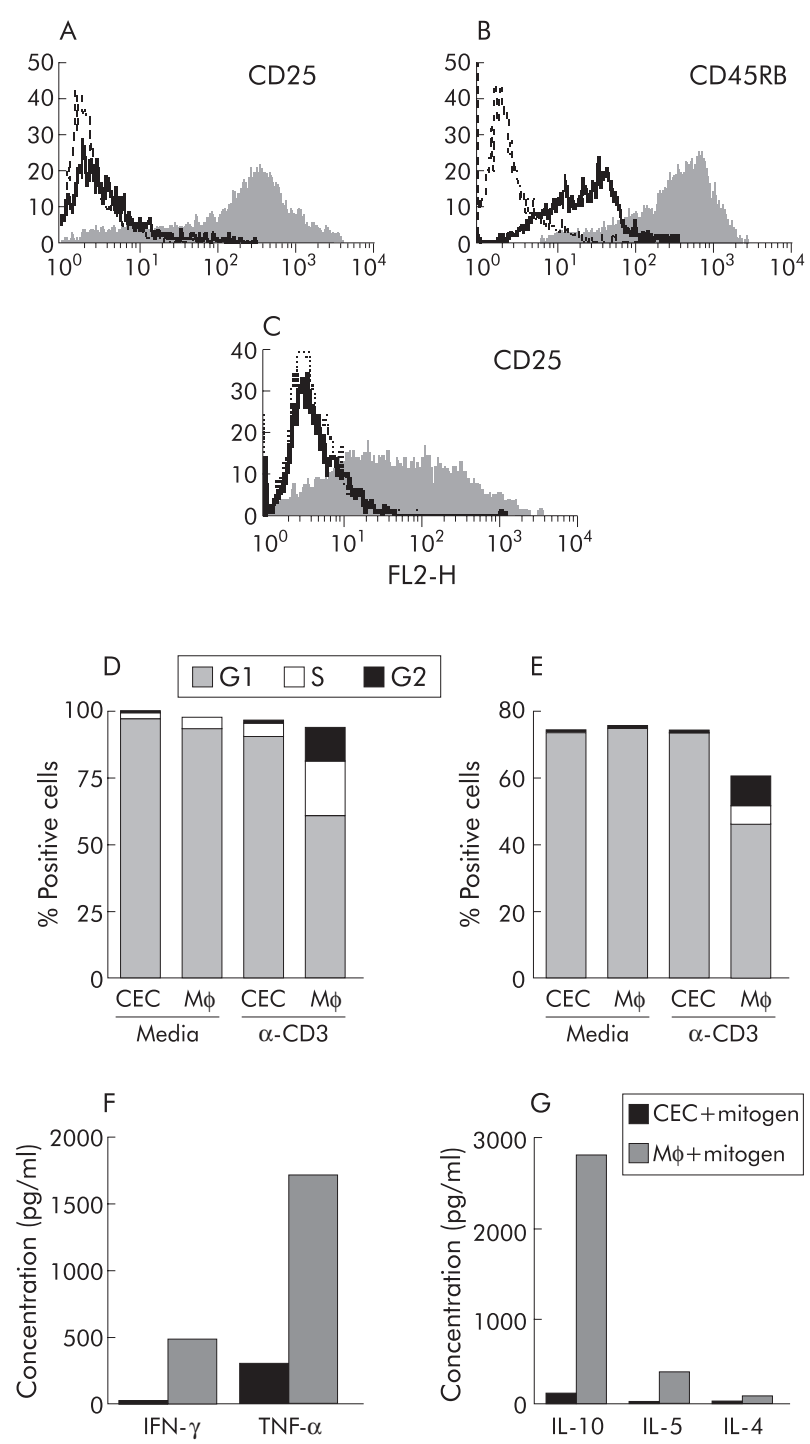

Figure 1 Colonic epithelial cells (CEC) inhibit the response of $T$ cells to conventional mitogens. Splenic CD4 T cells $(A, B, D, F, G)$ or colonic lamina propria CD4 T cells (C, E) were cultured with freshly isolated $\mathrm{C} 57 \mathrm{BL} / 6 \mathrm{CEC}$ or macrophages $(\mathrm{M} \phi)$ in the presence of mitogenic CD3 antibody for 72 hours prior to flow cytometric analysis for expression of CD25 on splenic (A) and lamina propria T cells (C), CD45RB on splenic CD4 T cells (B), cell cycle progression of splenic T cells (D) and lamina propria $T$ cells $(E)$, and cytokine production of splenic T cells using cytokine bead assays (F, G). In (A), (B), and (C), the filled in and solid line profiles represent levels of antibody staining of $T$ cells after coculture with $M \phi$ and CEC, respectively, and the broken lines represent the isotype matched control antibodies. (D, E) Proportion of splenic and lamina propria CD4 T cells in the G1, S, and G2 phases of the cell cycle. $(F, G)$ Cytokines produced by splenic T cells after culture with CEC and $M \phi$ in the presence of mitogen, respectively. Results are representative of those obtained from at least six independent experiments. IFN- $\gamma$, interferon $\gamma$; LL, interleukin; TNF- $\alpha$, tumour necrosis factor $\alpha$.

\section{CEC inhibit antigen specific CD4 T cell responses}

To assess if CEC could prime antigen specific T cells, CD4 cells from DO11.10 TcR transgenic mice, in which CD4 T cells express a TcR specific for a peptide of OVA and $\mathrm{H}-2^{\mathrm{d}}$, were cultured with OVA pulsed DO11.10 CEC. CEC were unable to prime CD4 T cells, as judged by lack of expression of CD25 and CD69 and absence of blast cell formation (data not shown).

CEC were unable to activate antigen primed $\mathrm{T}$ cells in a recall response. OVA primed CD4 cells cultured with $\mathrm{M} \phi$ or
CEC in the absence of antigen were quiescent, resided in the Gl phase of the cell cycle (fig 2C), and expressed low levels of CD25 (fig 2A), CD45RB (fig 2B), and Fas. Addition of antigen to cocultures of primed $\mathrm{T}$ cells and CEC did not induce $\mathrm{T}$ cell activation. CD4 cells remained quiescent, in the Gl phase of the cell cycle (fig 2C), with low expression of CD25 and CD45RB (fig 2A, B), and produced little or no IFN- $\gamma$, TNF- $\alpha$, or IL-10 (fig 2D). In contrast, primed CD4 cells cultured with $\mathrm{M} \phi$ and antigen expressed high levels of CD25 $(\mathrm{p}<0.005)$ and CD45RB $(\mathrm{p}<0.005)$, remained in the cell cycle (fig $2 \mathrm{~A})$, and produced cytokines, including IL-10 (fig 2D). Of note, CEC, were able to present antigen to the OVA specific CD4 hybridoma line $\mathrm{KZO}^{6}$ (fig 2E).

\section{CEC express costimulatory molecules}

The possibility that the inability of CEC to activate CD4 T cells was attributable to the absence of costimulation was investigated (fig 3) and compared with freshly isolated splenic $\mathrm{M} \phi$. All $\mathrm{M} \phi$ expressed CD54 with a high median fluorescence (MFI 149 (SD 22.47)) compared with 7.3\% CEC (MFI 10.54 (SD 3.8)). Up to $12 \%$ of M $\phi$ (MFI 10.62 (SD 0.66)) and 3\% CEC (MFI 9.5 (SD 4)) expressed B7.1. Seventy four per cent M $\phi$ expressed B7.2 (MFI 89.7 (SD 3.21 )), with a variable proportion of CEC (from $20 \%$ to $50 \%$ ) also expressing B7.2 (MFI 37.01 (SD 10)). Approximately 29\% M $\phi$ expressed CD40 (MFI 17.7 (SD 4)) compared with 4.5\% CEC (MFI 12.7 (SD 7.1)). Thus CEC expressed CD40 and B7.1 at levels comparable with those of splenic $\mathrm{M} \phi$ whereas the level of expression (MF) of B7.2 and CD54 was higher on $\mathrm{M} \phi$ than CEC. Expression of B7.1 and B7.2 was confirmed by reverse transcription-polymerase chain reaction and Southern blot analysis of CEC mRNA (Baumgart and Carding, unpublished observations). Of note, expression of costimulatory molecules on CEC was retained in culture.

\section{CEC are not inert and do not induce anergy in CD4 T cells}

The effects of increasing the threshold of T cell activation by addition of exogenous IL-2 $(200 \mathrm{U} / \mathrm{ml})$ or cross linking of CD28 on T cells which are known to overcome anergy were investigated. Addition of either IL-2 or anti-CD28 antibodies failed to restore T cell activation in the presence of CEC (fig 4). In contrast, addition of anti-CD28 antibody or IL-2 to $\mathrm{M} \phi: \mathrm{CD} 4 \mathrm{~T}$ cell cocultures potentiated the $\mathrm{T}$ cell response, increasing the proportion of cells in the cell cycle and levels of CD25 expression (fig 4). Of note, CD4 T cell responses to mitogen were inhibited by CEC, even in the presence of professional APC including $\mathrm{M} \phi$ (fig 4E), demonstrating that CEC are not inert and are actively suppressing or preventing CD4 $\mathrm{T}$ cell activation.

\section{Mechanisms of CEC mediated suppression of CD4 T cell activation}

The production of suppressive cytokines or soluble factors, the action of counterregulatory molecules of costimulation (for example, CTLA-4), or insufficient levels of MHC molecules were excluded as mechanisms of CEC mediated suppression of CD4 T cell activation. CEC conditioned media was unable to inhibit mitogen activation of CD4 cells by $\mathrm{M} \phi$, indicating that CEC mediated suppression was not due to the production of suppressive soluble factors, including IL-10 (figs 1G, 2D), and was cell contact dependent.

The possibility that CTLA-4 (CD152) was involved in CEC mediated suppression of $\mathrm{T}$ cell activation was excluded as CD4 cells in CEC cocultures were CTLA-4 negative although low levels of CTLA-4 were detected on the surface and cytoplasm of $\mathrm{T}$ cells from $\mathrm{M} \phi$ :CD4 T cell cocultures (data not shown). Cross linking of CD28 on T cells in the presence of 

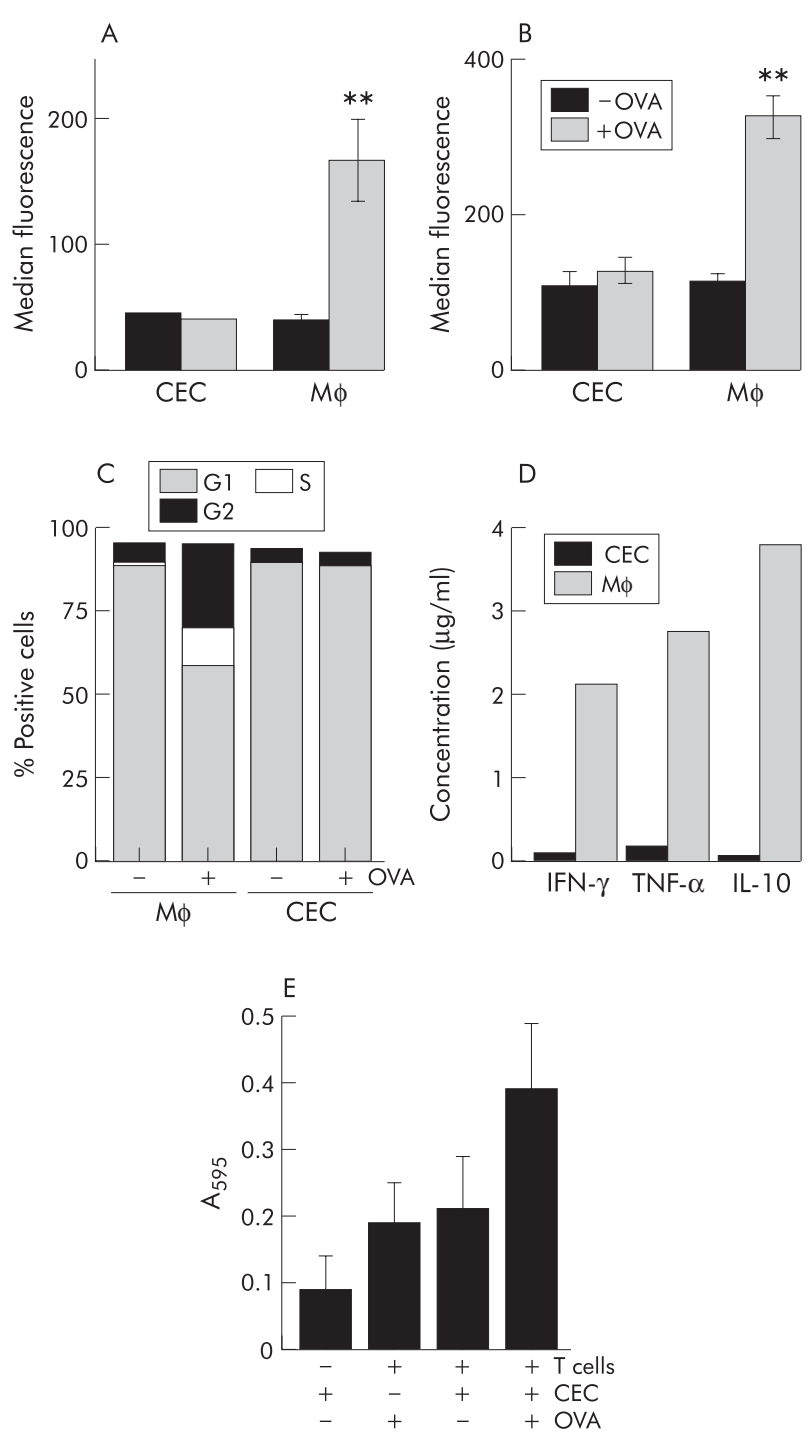

Figure 2 Responses of antigen specific T cells are inhibited by colonic epithelial cells (CEC). I-A ${ }^{\mathrm{d}}$-restricted DO11.10 CD4 T cells were cocultured with OVA primed DO1 1.10 CEC or macrophages $(M \phi)$ in the presence or absence of OVA for 72 hours prior to flow cytometric analysis of T cells for expression of CD25 (A), CD45RB (B), cell cycle progression (C), and cytokine production (D). (A, B) Cells cultured in the absence or presence of OVA: ${ }^{* *} p<0.005$ (A) and ${ }^{* *} p<0.005$ (B) comparing CD4 T cell activation in the presence of BALB/C (I-Ad) CEC or $M \phi$. (C) Proportion of CD4 T cells in the G1, S, and G2 phases of the cell cycle, respectively, with the plus and minus symbols referring to the presence or absence of OVA in the coculture. (D) Cytokine production by CD4 T cells cocultured with CEC and $M \phi$, respectively. Results are representative of those obtained from at least eight independent experiments. IFN- $\gamma$, interferon $\gamma$; IL, interleukin; TNF- $\alpha$, tumour necrosis factor $\alpha$. (E) C57BL/ 6 (I-A $)$ CEC can present OVA to an OVA specific I-A ${ }^{b}$ restricted CD4 T cell hybridoma KZO. Cultured CEC were pulsed with OVA, washed, and incubated for 24 hours with KZO, which had been transfected with an NFAT-lacZ reporter gene construct. T cell hybridoma activation and lacZ activity were determined spectrophotometrically using the chromogenic substrate and measuring absorbance at $595 \mathrm{~nm}$ (OD 595).

CEC (fig 4C, D) failed to restore T cell function, consistent with no role for B7.2, the ligand for CTLA-4, in CEC mediated suppression of $\mathrm{T}$ cells.

Increasing the level of MHC II on CEC was also unable to overcome CEC mediated suppression of T cells and CD4 T cell responses to mitogens (cross linking $\mathrm{CD} 3$ ), which are not
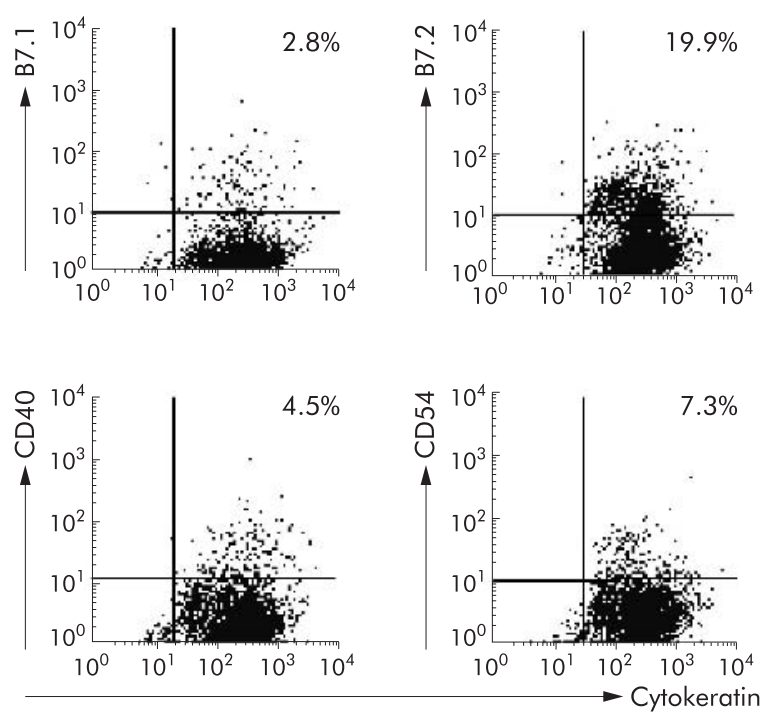

Figure 3 Colonic epithelial cells (CEC) express costimulatory molecules. Freshly isolated CEC were analysed by flow cytometry using antibodies specific for the epithelial cell specific marker cytokeratin and the costimulatory molecules B7.1 (CD80), B7.2 (CD86), CD40, and CD54. The quadrants were drawn based on isotype and single stained controls. Per cent values refer to the proportion of cells that were stained positive. Results are representative of those obtained from more than 10 independent experiments.

dependent on levels of MHC, were also inhibited in the presence of CEC (fig 1).

Although freshly isolated CEC expressed both cell surface associated TGF- $\beta$ (fig 5A) and latency associated peptide of TGF- $\beta$ (LAP; fig 5B), a neutralising antibody to TGF- $\beta 1-3$ $(20 \mu \mathrm{g} / \mathrm{ml})$ had no effect on CEC mediated suppression of CD4 $\mathrm{T}$ cell activation (fig 5C). This antibody was however able to block TGF- $\beta$ mediated inhibition of fibroblast proliferation (fig 5D). These experiments demonstrate that TGF- $\beta$ is not involved in CEC mediated suppression of T cells, either in soluble or cell surface bound form.

\section{Removal and restimulation of CD4 T cells from CEC cocultures renders them responsive}

CEC do not irreversibly suppress T cell activation. CD4 T cells recovered from CEC or $\mathrm{M} \phi$ cocultures and cultured with freshly isolated $\mathrm{M} \phi$ were activated by PMA/ionomycin or anti-CD3 antibody (fig 6B). Indeed, CD4 T cells previously cultured with CEC responded more vigorously than those previously cultured with $\mathrm{M} \phi$ to pharmacological challenge, with a greater proportion of cells entering the cell cycle (fig 6A). Thus CEC did not induce death of CD4 T cells, which was independently confirmed by failure to detect increased apoptosis by annexin $\mathrm{V}$ and propidium iodide staining (data not shown).

Antigen specific suppression of CD4 T cell activation was also reversible. DO11 T cells recovered from CEC cocultures containing antigen (OVA) and recultured with freshly isolated $\mathrm{M} \phi$ and OVA were activated, as assessed by cell cycle analysis and CD25 expression (fig 6C, D). The response of $\mathrm{T}$ cells previously cultured with CEC was of a slightly greater magnitude than those cells previously cultured with $\mathrm{M} \phi$. This may reflect activation induced cell death of T cells during the initial response to OVA in the presence of $\mathrm{M} \phi$. This contrasts with CD4 $\mathrm{T}$ cells previously cultured with CEC which remain in a resting state and have not undergone any significant apoptosis, thus providing a larger pool of responder $\mathrm{T}$ cells when restimulated with fresh $\mathrm{M} \phi$ and OVA. Taken together, these data demonstrates that CEC do 

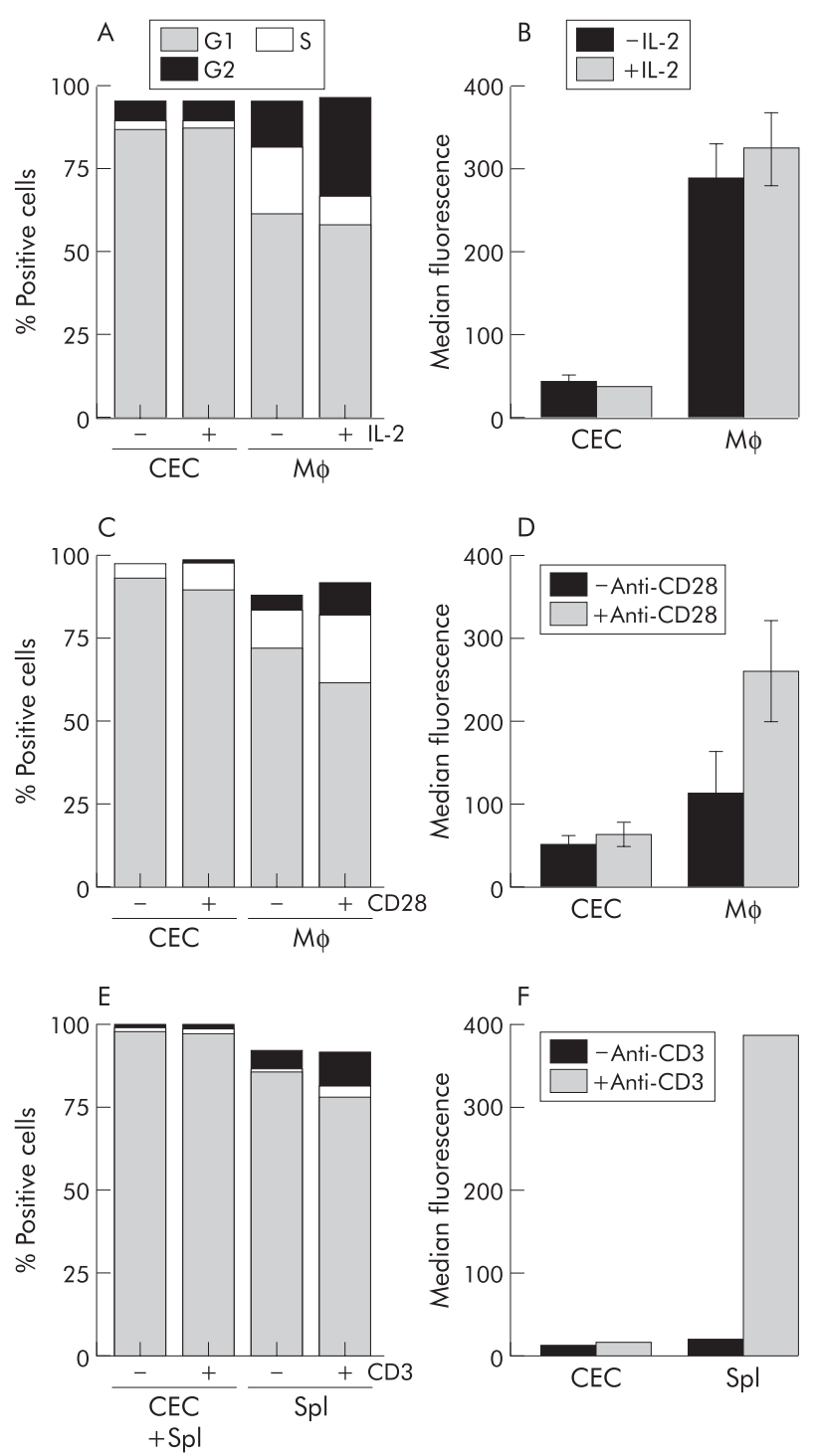

Figure 4 T cells cultured with colonic epithelial cells (CEC) are not anergised. CD4 T cells were cultured with freshly isolated murine CEC or macrophages $(M \phi)$ and mitogenic CD3 antibody in the presence $(+)$ or absence (-) of $200 \mathrm{U} / \mathrm{ml}$ interleukin 2 (IL-2) (A, B) or $10 \mu \mathrm{g} / \mathrm{ml} \mathrm{CD28}$ cross linking antibody (C, D). Whole spleen cells (Spl) were cultured in the presence or absence of CEC and a mitogenic anti-CD3 antibody $(E$, F). Activation was assessed by cell cycle analysis (A, C, E) and expression of the activation marker CD25 (B, D, F). (A, C, E) Proportion of CD4 T cells in the G1, S, and G2 phases of the cell cycle, respectively. The positive and minus symbols refer to the presence or absence of IL-2 $(A)$, anti-CD28 cross linking antibody $(C)$, and anti-CD3 antibody $(E)$, respectively. (B, D, F) Absence or presence of IL-2 (B), anti-CD28 antibody (D), and anti-CD3 antibody (F). Results are representative of those obtained from at least five independent experiments.

not permanently disable CD4 T cell function, with normal function being restored when CEC were removed.

\section{DISCUSSION}

Using a novel primary CEC culture system, we have shown that CEC, in contrast with splenic $M \phi$, suppress CD4 T cell activation. This inhibitory effect was dependent on the continual presence of CEC and was reversible. CEC mediated suppression of $\mathrm{T}$ cell responses may therefore play a role in maintaining non-responsiveness to enteric antigens. Although the experimental approach we have used is somewhat artificial, and fails to account for the cellular
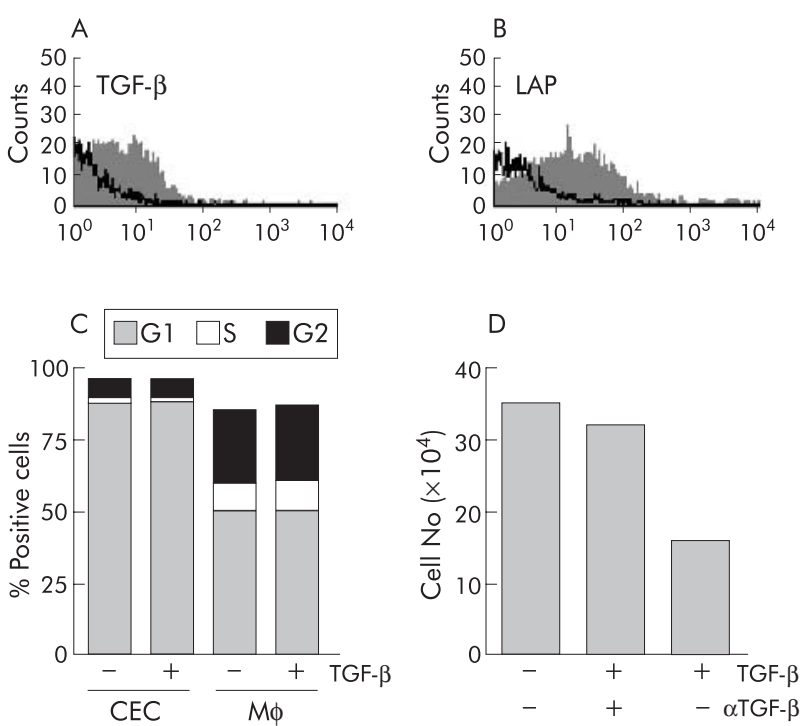

Figure 5 Colonic epithelial cells (CEC) mediated suppression of T cell responses is transforming growth factor $\beta$ (TGF- $\beta$ ) independent. Freshly isolated CEC were analysed by flow cytometry for expression of cell surface TGF- $\beta$ (A) and latency associated peptide (LAP) of TGF- $\beta$ (B). Filled histograms show antibody labelling compared with the isotype control (black line). (C) Effect of neutralising TGF- $\beta$ antibody $(20 \mu \mathrm{g} / \mathrm{ml})$ on cell cycle progression of CD4 cells in the presence of CEC or macrophages $(M \phi)$; proportion of CD4 T cells in the G1, S, and G2 phases of the cell cycle, and the plus and minus symbols refer to the presence or absence of the neutralising TGF- $\beta$ antibody in the coculture. (D) Effect of the neutralising antibody on proliferation of fibroblasts grown in Matrigel in the presence or absence of TGF- $\beta 1(100 \mathrm{ng} / \mathrm{ml})$. Results are representative of those obtained from at least four independent experiments.

heterogeneity of the colonic mucosa and possible influence of other mucosal cells on CEC function, it provides an in vitro accessible system with which to dissect colonic mucosal immune responses. The observation that CEC suppress activation of both naive and primed splenic as well as colonic LP CD4 T cells suggests that the suppressive effects of CEC are not restricted to resident mucosal $\mathrm{T}$ cells but also to cells from other tissues that may migrate to the colon.

Previously we have shown that murine small intestinal epithelial cells and CEC can present soluble protein antigen to antigen specific $\mathrm{CD}^{+} \mathrm{T}$ cell hybridomas. ${ }^{10}$ These findings conflict with our current observations that murine CEC inhibit the responses of primary CD4 T cells. This apparent discrepancy may be explained by the fact that $\mathrm{T}$ cell hybridomas and primary $\mathrm{T}$ cells have different requirements for activation, with $\mathrm{T}$ cell hybridomas having a lower threshold for activation and are costimulation independent. ${ }^{22}$ Although it has been shown that CEC can process antigen and present soluble protein antigens, ${ }^{1-6}$ primary $\mathrm{T}$ cells will not respond to antigen in the absence of costimulation. Our finding that murine CEC from two strains of mice constitutively expressed costimulatory molecules contrasts with a previous analysis of normal human CEC. ${ }^{19}$ This may reflect species differences or differences in the methods of detection as our analysis of murine CEC has shown that it was only possible to detect expression of B7.2 using a sensitive indirect staining procedure and was undetectable using direct methods (Cruickshank and Carding, unpublished observations).

The observation that CEC failed to promote mitogen or antigen specific responses despite expressing B7 suggests that their costimulatory molecules are not functional. The absence of CTLA-4 expression by T cells cultured with CEC excludes the involvement of this counter receptor. Also, the ability of 

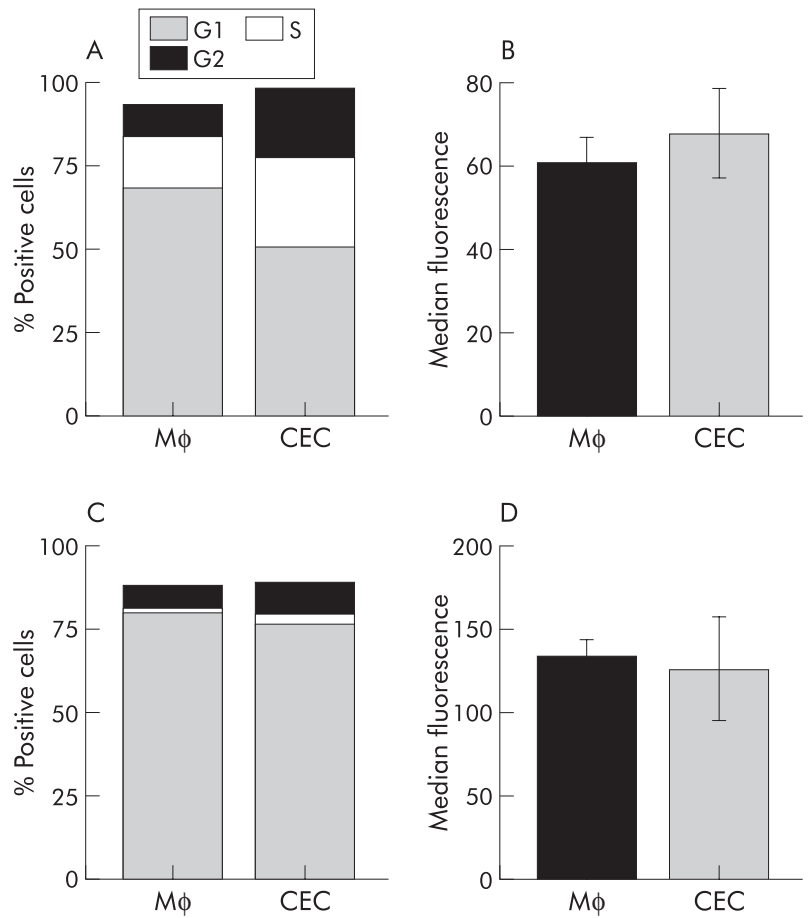

Figure $6 \mathrm{CD} 4$ cells previously cocultured with colonic epithelial cells (CEC) responded normally to restimulation. Naiive (A, B) or antigen primed $(C, D) C D 4$ cells were cocultured with CEC or macrophages $(M \phi)$ for 72 hours in the presence of mitogenic anti-CD3 antibody or OVA, after which they were isolated from cocultures and stimulated with phorbol-12-myristate 13-acetate (PMA)/ionomycin plus fresh $M \phi(A, B)$ or OVA pulsed $M \phi(C, D)$. T cell activation was assessed by cell cycle analysis $(A, C)$ and expression of the activation marker CD25 (B, D). (A, C) Proportion of CD4 T cells in the G1, S, and G2 phases of the cell cycle, respectively. (B, D) Expression of CD25 on CD4 T cells cultured with CEC or $M \phi$, respectively. Results shown are representative of those obtained from at least four independent experiments.

CEC to suppress $\mathrm{T}$ cell activation in the presence of splenic $\mathrm{M} \phi$ or anti-CD28 antibodies is consistent with the interpretation that CEC are not inert but actively suppress CD4 T cell activation. CEC do not anergise CD4 $\mathrm{T}$ cells as $\mathrm{T}$ cells previously cultured with CEC responded normally to rechallenge with mitogens or antigens. Thus the continued presence of the epithelial cell is needed for the suppressive effect. Similar suppressive effects have been reported for small IEC. ${ }^{23}$

In contrast with previous studies using non-viable (fixed) primary or established CEC lines, ${ }^{24-26}$ we have been unable to demonstrate that CD4 T cells cultured with CEC become T regulatory or suppressor cells. Although CD4 T cells cultured with murine CEC resembled Treg cells in being CD45RB ${ }^{\text {lo }}$ they were also CD25-lo , did not secrete IL-10, and responded normally to rechallenge by a variety of stimuli. Our data suggest therefore that CEC themselves are regulatory cells.

We were unable to identify the mechanism(s) by which CEC suppress $\mathrm{T}$ cell activation. The observation that supernatants taken from growing cultures of CEC had no effect on $\mathrm{T}$ cell activation are consistent with the effect being cell and probably contact dependent. However, the possibility that suppression is mediated by a soluble factor that is only produced when $\mathrm{T}$ cells are present, or only acts when cells are in close proximity, cannot be excluded. Although a recent publication has ascribed a role for cell surface bound TGF- $\beta$ in mediating the suppressive function of regulatory $\mathrm{T}$ cells, ${ }^{27}$ our data show that TGF- $\beta$ is not involved in CEC mediated suppression of CD4 T cell activation. Potential mechanisms include mucins and novel inhibitory costimulatory molecules. The mucin associated molecule MUC-1 has been reported as having suppressive or proapoptotic effects on $\mathrm{T}$ cell activation 2829 and murine CEC expressed MUC-1 (Cruickshank and Carding, unpublished data). The lack of suitable reagents however prevents us from determining what role, if any, it might play in CEC mediated suppression of CD4 T cells. The recent observations that novel members of the $\mathrm{B} 7$ family $\left(\mathrm{B}_{\mathrm{X}},{ }^{30}\right.$ and the ligands for PD-1; B7-Hl (PD-L1) and PD-L2 ${ }^{31}$ ) have inhibitory effects on $\mathrm{T}$ cell activation may also provide an alternative mechanism for CEC mediated suppression of $\mathrm{T}$ cell activation although it is not known if CEC express these negative receptors.

In conclusion, we have demonstrated that normal murine CEC actively suppress CD4 $\mathrm{T}$ cell responses and that this effect is reversible. We believe that CEC, as the major site of antigen contact in the colon, play a role in preventing the underlying $\mathrm{T}$ cells in the stroma from responding to enteric antigens, which can be presented by epithelial cells. Our findings also may explain how global non-responsiveness of mucosal $\mathrm{T}$ cells to the vast array of enteric antigens sampled by CEC is maintained. Dysregulation of CEC mediated suppression of $\mathrm{T}$ cell responses may also contribute to the uncontrolled inflammation seen in inflammatory bowel diseases.

\section{ACKNOWLEDGEMENTS}

This work was supported by Public Health Service grants AI-41562 and POlRR12211, (SR Carding, PJ Felsburg)

\section{Authors' affiliations}

S M Cruickshank, S R Carding, School of Biochemistry and Molecular Biology, University of Leeds, Leeds, UK

L D McVay, Harrison Department of Surgical Research, University of Pennsylvania School of Medicine, Philadelphia, PA, USA

D C Baumgart, Universitaestklinikum Charite der Humboldt-Universitaet zu Berlin, Campus Rudolf Virchow-Klinikum, Medizinische Klinik mit Schwerpunkt, Hepatologie und Gastroenterologie, Berlin, Germany P J Felsburg, Department of Clinical Studies, University Pennsylvania School of Veterinary Medicine, Philadelphia, PA, USA

\section{REFERENCES}

1 Shao L, Serrano D, Mayer L. The role of epithelial cells in immune regulation in the gut. Semin Immunol 2001;13:163-76.

2 Mayer L, Eisenhardt D, Salomon P, et al. Expression of class II molecules on intestinal epithelial cells in humans. Differences between normal and inflammatory bowel disease. Gastroenterology 1991;1191:100-3.

3 Hershberg RM, Cho DH, Youakim A, et al. Highly polarized HLA class II antigen processing and presentation by human intestinal epithelial cells. J Clin Invest 1998;102:792-803.

4 Blumberg RS, Lencer WI, Zhu X, et al. Antigen presentation by intestinal epithelial cells. Immunol Lett 1999;69:7-11.

5 Campbell N, Yio XY, So LP, et al. The intestinal epithelial cell: processing and presentation of antigen to the mucosal immune system. Immunol Rev 1999;172:315-24.

6 Mayer L, Blumberg RS. Antigen presenting cells; Epithelial cells. In:Ogra PL, Mestecky J, Lamm ME, et al, editors. Mucosal immunology. San Diego: Academic Press, 1999:365-379.

7 Garside P, Mowat AM. Oral tolerance. Semin Immunol 2001:13:177-85.

8 Strober W, Fuss IJ, Blumberg RS. The immunology of mucosal models of inflammation. Ann Rev Immunol 2002;20:495-549.

9 Kato T, Owen RL. Structure and function of intestinal mucosal epithelium. In:Ogra PL, Mestecky J, Lamm ME, et al, editors. Mucosal immunology. San Diego: Academic Press, 1999:115-32.

10 Telega GW, Baumgart DC, Carding SR. Uptake and presentation of antigen to $T$ cells by primary colonic epithelial cells in normal and diseased states. Gastroenterology 2000;1 19:1548-59.

11 Carreno BM, Collins M. The B7 family of ligands and its receptors: new pathways for costimulation and inhibition of immune responses. Ann Rev Immunol 2002;20:29-53.

12 Leon M, Kirby J, Gibbs P, et al. Immunogenicity of biliary epithelial cells: study of the expression of B7 molecules. J Hepatol 1995;22:591-6.

13 Ye G, Barrera C, Fan X, et al. Expression of B7-1 and B7-2 costimulatory molecules by human gastric epithelial cells: potential role in CD4+ T cell activation during Helicobacter pylori infection. J Clin Invest 1997;99:1628-36. 
14 Cruickshank SM, Southgate J, Selby PJ, et al. Expression and cytokine regulation of immune recognition elements by normal human biliary epithelial and established liver cell lines in vitro. J Hepatol 1998;29:550-8.

15 Leon MP, Bassendine MF, Wilson JL, et al. Immunogenicity of biliary epithelium: Investigation of antigen presentation to CD4+ T cells. Hepatology 1996;24:561-7.

16 Cunningham AC, Zhang JG, Moy JV, et al. A comparison of the antigenpresenting capabilities of class II MHC-expressing human lung epithelial and endothelial cells. Immunology 1997;91:458-63.

17 Cruickshank SM, Southgate J, Selby PJ, et al. Inhibition of T cell activation by normal human biliary epithelial cells. J Hepatol 1999:31:1026-33

18 Spengler U, Leifeld L, Braunschweiger I, et al. Anomalous expression of costimulatory molecules B7-1, B7-2 and CD28 in primary biliary cirrhosis. J Hepatol 1997;26:31-6.

19 Nakazawa A, Watanabe M, Kanai T, et al. Functional expression of costimulatory molecule CD86 on epithelial cells in the inflamed colonic mucosa. Gastroenterology 1999;117:536-45.

20 Baumgart DC, Olivier WA, Reya T, et al. Mechanisms of intestinal epithelial cell injury and colitis in interleukin 2 (IL2)-deficient mice. Cell Immunol 1998; 187:52

21 Ebert EC. Proliferative responses of human intraepithelial lymphocytes to various T-cell stimuli. Gastroenterology 1989;97:1372-81.

22 Sanderson S, Shastri N. LacZ-inducible, antigen/MHC specific T cell hybrids. Int Immunol 1994;6:369-76.
23 Bland PW, Warren LG. Antigen presentation by epithelial cells of the rat small intestine. I. Kinetics, antigen specificity and blocking by anti-la antisera. Immunology 1986;58:1-7.

24 Arosa FA, Irwin C, Mayer L, et al. Interactions between peripheral blood CD8 T lymphocytes and intestinal epithelial cells (iEC). Clin Exp Immunol 1998;112:226-36.

25 Allez M, Brimnes J, Dotan I, et al. Expansion of CD8+ T cells with regulatory function after interaction with intestinal epithelial cells. Gastroenterology 2002;123:1516-26.

26 Bland PW, Warren LG. Antigen presentation by epithelial cells of the rat small intestine. II. Selective induction of suppressor T cells. Immunology 1986;58:9-14.

27 Nakamura K, Kitani A, Strober W. Cell contact-dependent immunosuppression by $\mathrm{CD} 4(+) \mathrm{CD} 25(+)$ regulatory T cells is mediated by cell surface-bound transforming growth factor beta. J Exp Med $2001 ; 194: 629-44$

28 Chang JF, Zhao HL, Phillips J, et al. The epithelial mucin, MUC1, is expressed on resting $T$ lymphocytes and can function as a negative regulator of $\mathrm{T}$ cell activation. Cell Immunol 2000;201:83-8.

29 Gimmi CD, Morrison BW, Mainprice BA, et al. Breast cancer-associated antigen, DF3/MUC1, induces apoptosis of activated human T cells. Nature Med 1996;2:1367-70.

30 Zang $\mathrm{X}$, Loke $\mathrm{P}, \mathrm{Kim}$ J, et al. B7x: A widely expressed B7 family member that inhibits T cell activation. Proc Natl Acad Sci U S A 2003;100:10388-92.

31 Loke P, Allison JP. PD-L1 and PD-L2 are differentially regulated by Th1 and Th2 cells. Proc Natl Acad Sci U S A 2003;100:5336-41. 